\title{
Indirect Inactivation of Candida guilliemondii by Using a Plasma Synthetic Jet Actuator: Effect of Advected Charged Particles
}

\author{
Gabriele Neretti, a, ${ }^{a}$ Anna C. Ricchiuto, ${ }^{a}$ Roberta Galuppi, ${ }^{b}$ Giovanni Poglayen, ${ }^{b}$ \\ Benedetto Morandi, ${ }^{b}$ Ester Marotta, ${ }^{c}$ Cristina Paradisi, ${ }^{c}$ Francesco Tampieri, ${ }^{c}$ \\ \& Carlo A. Borghia \\ aElectrical, Electronic and Information Engineering Department, University of Bologna, Italy;

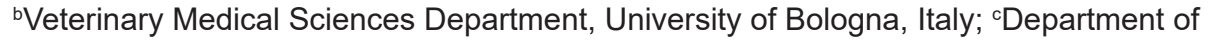 \\ Chemical Sciences, University of Padova, Italy \\ *Address all correspondence to: G. Neretti, Electrical, Electronic and Information Engineering Department, University \\ of Bologna, Italy, Viale del Risorgimento, 2, 40136, Bologna, Italy, E-mail: gabriele.neretti@unibo.it
}

\begin{abstract}
Plasma synthetic jet actuators (PSJAs) demonstrated their ability to produce a tubular flow normal to the surface where the dielectric barrier discharge (DBD) is ignited. These fluid dynamics actuators enhance the delivery of reactive and charged species toward the target to be treated. In this work, the efficacy of an octagonal PSJA used to indirectly treat Candida guilliermondii, both in agar substrate and in saline solution, was experimentally studied. The inactivation effect of free charges advected by the induced flow has been investigated as well by interposing a metallic mesh between the actuator surface and the treated sample. Therefore, the passage of charges and their migration toward the sample can either be allowed or prevented simply by having the mesh floating or connected to the ground, respectively. A $4 \log$ and a 2 log colony-forming unit (CFU) reduction were observed for agar substrate and saline solution, respectively, at the highest energy dose treatment with the grid grounded. Under the same conditions but with the grid at floating potential, the presence of advected charges gives an additional $1 \log$ CFU. In addition to biological tests, chemical analyses have been carried out to identify the most important modifications produced by the induced flow into treated water. Results presented in this work demonstrate the efficacy of PSJAs in indirect treatment of C. guilliermondii, both on agar substrate and saline solution, and the inactivation properties of free charges advected by this type of fluid dynamics actuator.
\end{abstract}

KEY WORDS: nonthermal plasma, fluid dynamics, charged particles, indirect treatment, Candida

\section{INTRODUCTION}

Candida species belong to the normal microbiota of mucous membranes in vertebrates and may cause systemic infection in an immunocompromised host. In particular, in humans, species of the genus Candida are the agents most frequently implicated in invasive nosocomial fungal infections as a consequence of bone marrow and solid organ transplantation, chemotherapy, and immunosuppression. More than 30 species of Candida have been reported to cause invasive candidiasis. ${ }^{1}$ Candida albicans is usually the predominant species found. In a European survey on candidemia, it represented about 
$56 \%$ of isolates; ${ }^{2}$ however, non-albicans Candida (NAC) were the most frequently isolated from patient with hematological malignance (65\%), confirming the importance of these species as causes of life-threatening nosocomial infection. Among NAC species, Candida guilliermondii is widely distributed in nature and frequently isolated from soil, plants, insects, seawater, atmosphere, exudates of several trees, and processed foods, in addition to being a part of saprophyte microflora on the skin and mucosal surfaces of humans $^{3}$ and also of animals. ${ }^{4}$ Although it is a rare cause of invasive candidiasis in humans and shows a reduced innate virulence compared with $C$. albicans, $C$. guilliermondii has aroused interest due to its intrinsic resistance to some antifungal drugs. ${ }^{3,5,6}$

In recent years, cold atmospheric pressure plasmas are emerging as a promising technology into the biomedical domain. ${ }^{7}$ The main features of these non-thermal discharges are their low operational cost due to operation at room temperature and pressure and virtual absence of residual and toxic emissions. Several works demonstrated the sterilization ability of cold plasma against bacteria, viruses, yeasts. and parasites. ${ }^{8-11}$ Direct and indirect treatments demonstrate the inactivation ability of cold atmospheric pressure plasmas against Candida albicans too, ${ }^{12-19}$ but to the authors' knowledge, no information exists about $C$. guilliermondii treatment. In direct plasma treatment, the surface of pathogens is exposed to reactive oxygen and nitrogen species, UV radiation, electric fields, and a significant flux of charged particles, including electrons and positive and negative ions. ${ }^{20,21}$ Usually, in indirect treatments, only sufficiently long-lived reactive species are considered to be effective in the inactivation process. In direct treatments, more "actors" play together in a synergic way, contributing to the sterilization effect. Nevertheless, direct treatments are not always the best solution, especially if irregular and large area surfaces must be treated. Moreover, the direct plasma application could create unwanted modifications in the physical and chemical composition of the treated sample (organoleptic food properties as an example). Lastly, indirect treatments allow the plasma reactor to be developed and optimized without considering the target to be treated.

To expand the range of possible indirect treatments, our research group recently developed a plasma synthetic jet actuator (PSJA) able to produce an ionic wind normal to the surface where the dielectric barrier discharge (DBD) is ignited. ${ }^{22}$ This jet propagates for about $10 \mathrm{~cm}$ at a velocity of several meters per second, enhancing the delivery of reactive species onto a target to be treated. ${ }^{23}$ The production of the ionic wind is due to the electro-hydro-dynamic (EHD) interaction. In a surface DBD (SDBD) ions are produced within the plasma region and then accelerated by the applied electric field. These heavily charged particles collide with surrounding neutral ones, releasing momentum to them and producing a unidirectional body force parallel to the actuator surface. ${ }^{24-27}$ Actuators featured by annular geometries have shown the ability to produce a jet normal to the actuator surface. ${ }^{28}$ These fluid dynamics plasma actuators are now extensively studied for several applications in aeronautics and astronautics, ${ }^{29-33}$ and in the turbine blade domain. ${ }^{34,35}$ Basics physics studies on linear aerodynamics plasma actuators showed the presence of charges deposited onto the actuator surface at a distance up to several centimeters downstream the plasma region. These charges are 
produced within the plasma volume and subsequently advected by the tangential wall jet. ${ }^{36,37}$ A recent experimental work ${ }^{38}$ showed that annular actuators can also transport and deposit positive charges over a target positioned at several centimeters from the surface where the discharge is ignited.

The clinical potential of the charged particles onto biological substrates has been already studied both in direct and semi-indirect treatments. ${ }^{39-42}$ The aim of the present work was to verify the efficacy of a PSJA used to treat $C$. guilliermondii indirectly both in agar substrate and in water solution. The inactivation effect of free charges advected by the induced flow has been investigated as well. Finally, a preliminary chemical analysis was carried out to underline the effect of charged particles into the treated water.

\section{MATERIALS AND METHODS}

The PSJA utilized in this work is represented in Fig. 1 and it is the same described in Neretti et al. ${ }^{38}$ The upper octagonal electrode was connected to the high voltage source, the lower one was grounded. A metal mesh was positioned $1 \mathrm{~cm}$ from the actuator surface (in the " $y$ " direction) to give the possibility to arrest free charges advected by the tubular induced flow. This mesh has square holes with $2 \mathrm{~mm}$ sides and a wire thickness of $0.5 \mathrm{~mm}$.

A sinusoidal supply system constituted by a push-pull high-voltage transformer controlled by Arduino has been utilized to feed the discharge. It allows to change both voltage and frequency in the range $0-20 \mathrm{kVp}$ and $15-50 \mathrm{kHz}$, respectively, and can ignite the plasma with an arbitrary duty cycle. For the actuator considered in this work, a sinusoidal voltage of $6 \mathrm{kV}$ peak with a frequency of $31 \mathrm{kHz}$ was chosen. These parameters lead to an average power supplying the plasma equal to $14 \mathrm{~W}$. Electrical measurement setup is the same utilized in Neretti et al. ${ }^{38}$

The effect of the mesh into the fluid dynamics of the induced flow has been investigated by means of the Schlieren technique with a Z-type configuration. The

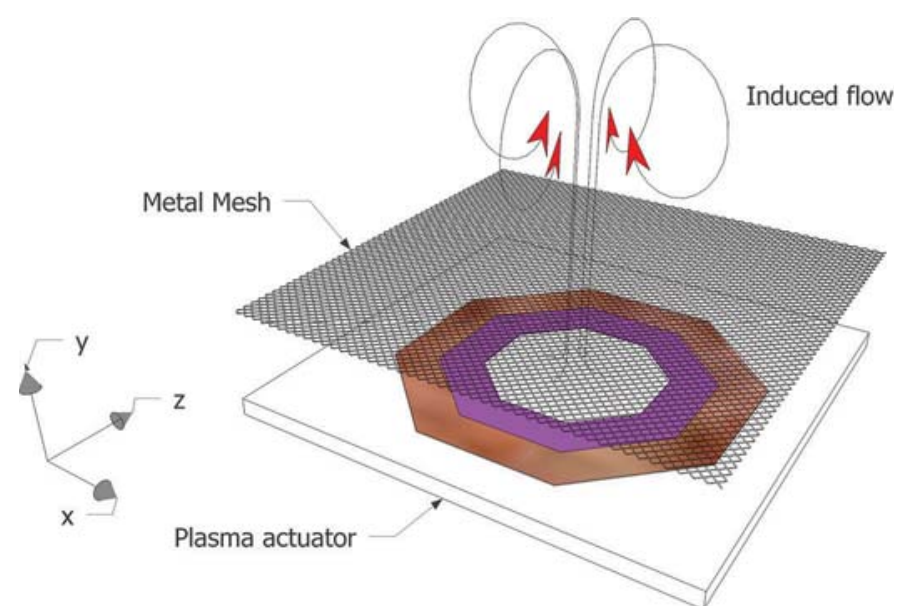

FIG. 1: PSJA sketch and metallic mesh used to arrest free charges

Volume 8, Issue 3, 2018 
experimental setup used in this work is the same described in Neretti et al. ${ }^{43}$ Conversely, the ability of the metallic mesh to arrest advected charges has been verified by using the same methodology described in Neretti et al. ${ }^{38}$ Charge distribution was evaluated from surface potential measurements by using the numerical approach described previously. ${ }^{36}$

The PSJA described above has been used to perform an indirect treatment on a strain of $C$. guilliermondii isolated from mare endometritis. The strain was maintained through serial transplants on Sabouraud dextrose agar (DIFCO) with $0.05 \%$ chloramphenicol. An $80 \mathrm{~mL}$ suspension of 48-hour aged colonies of the yeast in saline was obtained $(0.5$ opacity by McFarland's scale). To quantify the living yeast present, the number of colony-forming units (CFUs) of untreated suspension was determined performing multiple dilution that were plated on Sabouraud dextrose agar (SDA) Petri dishes and incubated at $26^{\circ} \mathrm{C}$ for 48 hours. Aliquots of $100 \mu 1$ of suspension were spread on the surface of 6 SDA plates ( $9 \mathrm{~cm}$ diameter); $20 \mathrm{~mL}$ aliquots of suspension were put into $69 \mathrm{~cm}$ plates. These samples (both on agar substrate and in saline suspension) have been treated using the setup reported in Fig. 2. The PSJA was mounted at a distance of $2 \mathrm{~cm}$ from the agar or water surface, with the metallic mesh connected either to the ground terminal or left floating. In this way, free charges have been blocked or left free to reach the sample. respectively. Electrostatic simulations preformed with FEMM software ${ }^{44}$ demonstrate that the presence of the mesh (grounded or left floating) does not influence the electric field distribution within the plasma formation region. Treatment times have been set to 0.5 , 2, and $5 \mathrm{~min}$ for agar substrate treatments and 5, 10, and $15 \mathrm{~min}$ for saline suspension. Plasma has been operated in discontinuous mode with equal on and off times of $1 \mathrm{sec}$. In this way, fluid dynamics of the jet can completely develop within the Petri dish, delivering reactive and charged species onto the whole Petri dish surface. By alternating these plasma-on time intervals with a $1 \mathrm{sec}$ plasma-off period, translational temperature of both discharge and dielectric surface are maintained low and a lower amount of energy dose is utilized to treat the sample.

After treatments, the CFU from each saline suspension was determined by multiple dilution as described previously; whereas, in the agar-treated plates, the colonies were directly counted after incubation at $26^{\circ} \mathrm{C}$ for 48 hours.

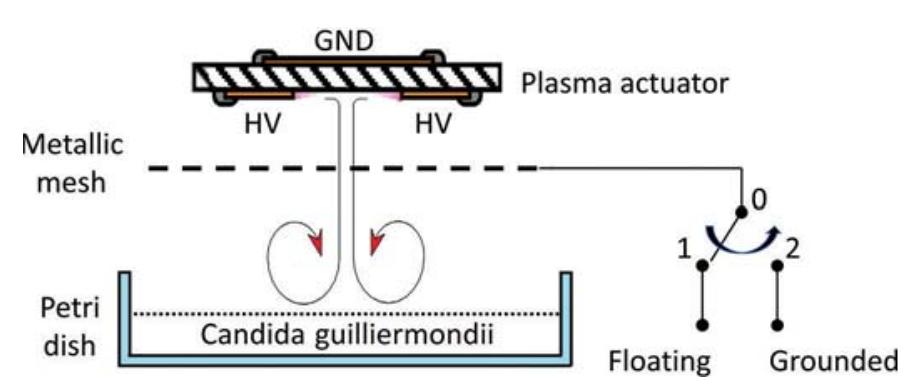

FIG. 2: Treatment setup sketch 
Chemical analysis was carried out to detect more important species dissolved and produced into plasma-treated water. Coumarin-3-carboxylic acid (97\%), 7-hydroxycoumarin carboxylic acid (98\%), potassium indigotrisulfonate, and titanium (IV) oxysulphate sulfuric acid solution (27-31\%) were purchased from Sigma-Aldrich. Ultrapure grade water (Milli-Q water) was obtained by filtration of deionized water with a Millipore system. To quantify the species produced in solution by the plasma source, we treated $10 \mathrm{~mL}$ aliquots of Milli-Q water inside a Petri dish for different lengths of time. For the determination of $\mathrm{OH}$ radicals, a $10^{-3} \mathrm{M}$ solution of coumarin-3-carboxylic acid was used instead of Milli-Q water. All of the experiments were repeated at least twice and the results averaged. The reproducibility was usually within $10 \%$. The $\mathrm{pH}$ of the solutions before and after plasma treatment was measured using a Mettler-Toledo S220 pH meter equipped with an InLab Versatile Pro electrode. The concentration of nitrite and nitrate ions in solution was measured by ion chromatography using a Dionex-ICS-900 instrument equipped with a Dionex IonPac AS22 IC Column after proper calibration with standard solutions. Then, $4.5 \mathrm{mM}$ sodium carbonate and $1.4 \mathrm{mM}$ sodium bicarbonate were used as eluent at a flow rate of $1.2 \mathrm{~mL} \mathrm{~min}^{-1}$. The concentration of ozone and hydrogen peroxide in solution was determined by the indigo ${ }^{45}$ and the titanium (IV) sulfate ${ }^{46}$ methods, respectively. The rate of formation of hydroxyl radicals was determined using coumarin-3-carboxylic acid as a fluorescent probe. ${ }^{47}$ All of the procedures have been described in detail previously. ${ }^{48-50}$

\section{RESULTS AND DISC USSION}

As already mentioned, the PSJA used in this work has been characterized previously. ${ }^{38}$ In the present study, a metallic mesh positioned at a distance $1 \mathrm{~cm}$ from the actuator surface has been added to give the possibility to arrest charges flowing toward the treated sample, allowing us to determine the influence of these charged particles in the inactivation process. All tests were carried out in open air with a relative humidity of $41 \pm 5 \%$ and a temperature of $25 \pm 2{ }^{\circ} \mathrm{C}$. A first series of experiments has been carried out to point out the influence of the mesh on the fluid dynamics of the induced jet and on the surface distribution of deposited charges.

Figure 3 shows a comparison of Schlieren images of the induced jets generated by the PSJA actuator without (Fig. 3a) and with (Fig. 3b) the metallic mesh recorded after 10,30 , and $60 \mathrm{~ms}$ from discarge ignition. The presence of the mesh influences the induced jet morphology and speed in a negligible way. When the mesh is added, especially in the image captured after $10 \mathrm{~ms}$ from discharge ignition, a "comb" spreading of the jet is clearly visible suddenly downstream of the metallic grid.

The influence of the metallic mesh into charges deposited over the target surface has been detected by following the same procedure described previously. ${ }^{38}$ The discharge was ignited for increasing time intervals, covering the whole treatment range from $30 \mathrm{sec}$ to $15 \mathrm{~min}$. Plasma was operated with the same duty cycle of $1 \mathrm{sec}$ "on" and $1 \mathrm{sec}$ "off" utilized in the treatments. The charge distribution detected for all treatment times was the same within a standard deviation error of $6 \%$. This result is explicable

Volume 8, Issue 3, 2018 

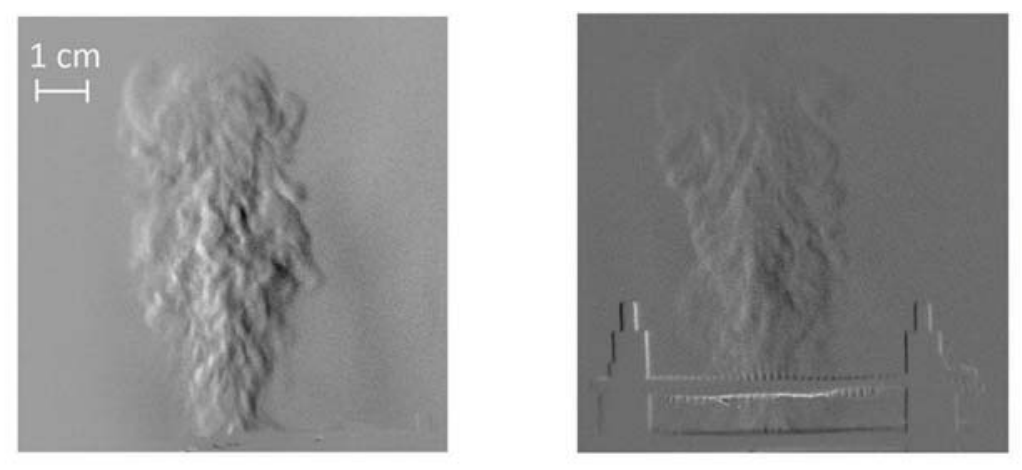

$60 \mathrm{~ms}$
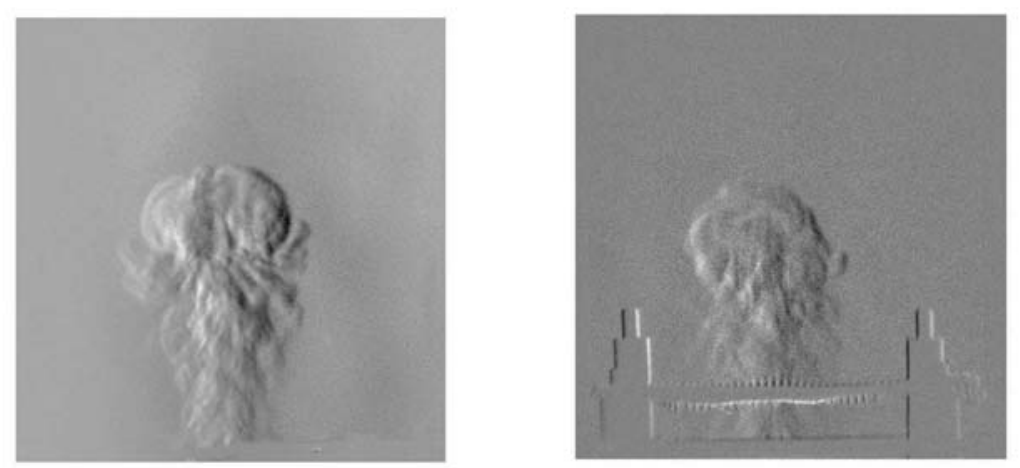

$30 \mathrm{~ms}$
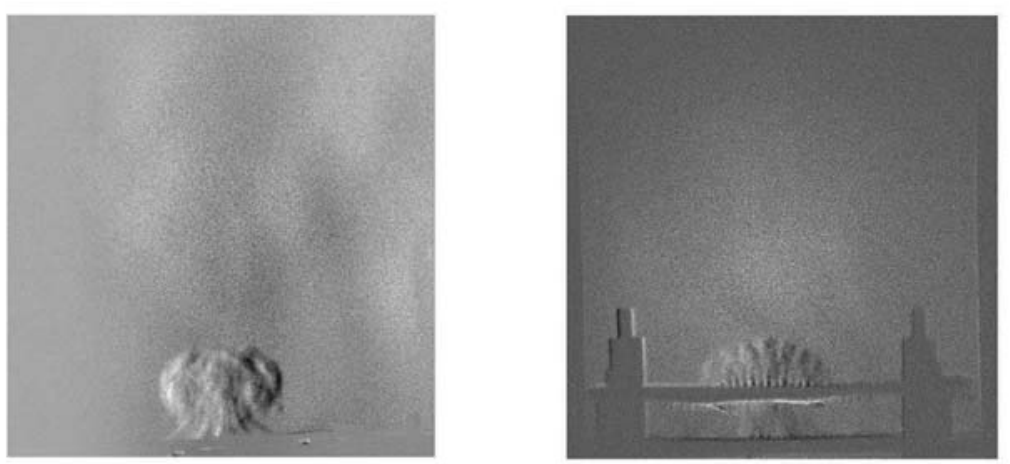

\section{$10 \mathrm{~ms}$}

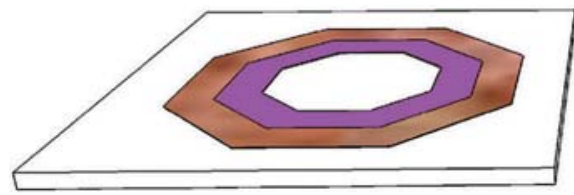

a) Without mesh

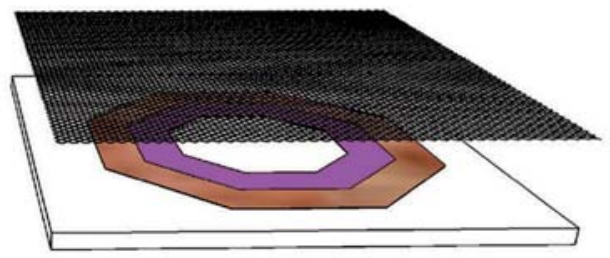

b) With mesh

FIG. 3: Schlieren images of the induced jets generated by the PSJA actuator without (a) and with (b) the metallic mesh after 10,30, and $60 \mathrm{~ms}$ from discarge ignition 
with the relatively fast dynamics with which charges are deposited over the target surface. Measurements reported previously ${ }^{38}$ show that after $500 \mathrm{~ms}$ of plasma on time, a quasi-steady-state in the charge deposition is achieved. Surface potential distribution and charge density measured after $30 \mathrm{~s}$ of plasma ignition are shown in Fig. 4. The probe position equal to $0 \mathrm{~mm}$ corresponds to the target position in front of the jet core. Charge distribution is not drastically modified when comparing the condition without mesh (red continuous line) or the one with floating mesh (black dashed line). When the mesh is present a more uniform charge distribution is obtained into the jet core. This is consistent with the tendency of the metallic mesh to create an isopotential surface. Conversely, "wings" observed in the charge distribution obtained without the mesh seems to disappear. This behavior is not easy to explain and deserves more investigation. When the mesh is grounded (magenta line with plus signs), about $90 \%$ of the advected charges are drained to the ground terminal, strongly limiting the number of charged particles reaching the target.

Inactivation results of $C$. guilliermondii obtained both in agar substrate (red color with circle symbols) and water solution (blue color with diamond symbols) are shown in Fig. 5. All treatments have been performed with the metal mesh left floating (continuous line) or grounded (dashed line). Treatments in agar have been repeated in triplicate. Error bars underline the consistency of results.

When positive charges are left free to reach the sample, a stronger plasma inactivation effect is achieved. As far as the average power is the same in all conditions, this additional inactivation effect is energy dose dependent, reaching an additional $1 \mathrm{log}$ reduction in CFU for longest treatment times. C. guilliermondii $\mathrm{CFU}$ reduction in agar substrate is subjected to a $\log 3$ decrement after a treatment of $30 \mathrm{sec}$, reaching complete inactivation after $5 \mathrm{~min}$ of treatment with a $5 \log$ reduction. Candida inactivation in water needs longer treatment times. After a 15 -min treatment about a $\log 3$ reduction has been achieved. Longer inactivation times for water treatments can be ascribed to longer times needed for plasma products to reach Candida cells. These products hit water sur-

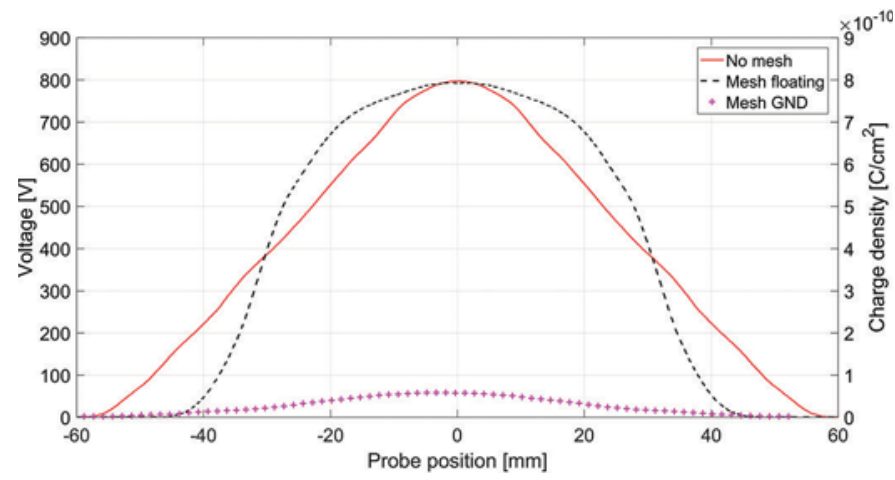

FIG. 4: Surface potential distribution and charge density measured over the dielectric target slab positioned at $2 \mathrm{~cm}$ from the actuator surface without mesh (continuous line), mesh left floating (dashed line), and mesh grounded (plus symbols)

Volume 8, Issue 3, 2018 


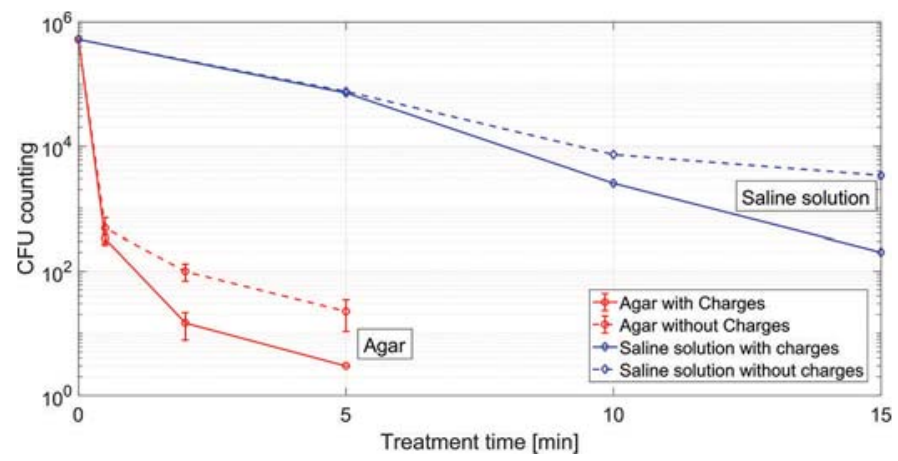

FIG. 5: CFU reduction performed on C. guilliermondii in agar substrate (circle symbols) and water solution (diamond symbols) with (continuous line) and without charges (dashed line)

face and then diffuse into the liquid volume, triggering the inactivation process. In the agar substrate condition, Candida cells are directly in contact with plasma products and the inactivation process takes place in shorter times.

It is important to point out that although the induced tubular jet core only has a diameter of about $1 \mathrm{~cm}$ (Fig. 3) and thus an area of $0.8 \mathrm{~cm}^{2}$, by hitting the target, it spreads over it, allowing a homogeneous inactivation effect over the whole Petri dish surface of $64 \mathrm{~cm}^{2}$. As a matter of fact, the Petri diameter is $9 \mathrm{~cm}$, about the same length (and thus respective surface) over which charges are deposited by the induced jet and reactive species are also transported (see curves where the mesh is present in Fig. 4). In Fig. 6, this

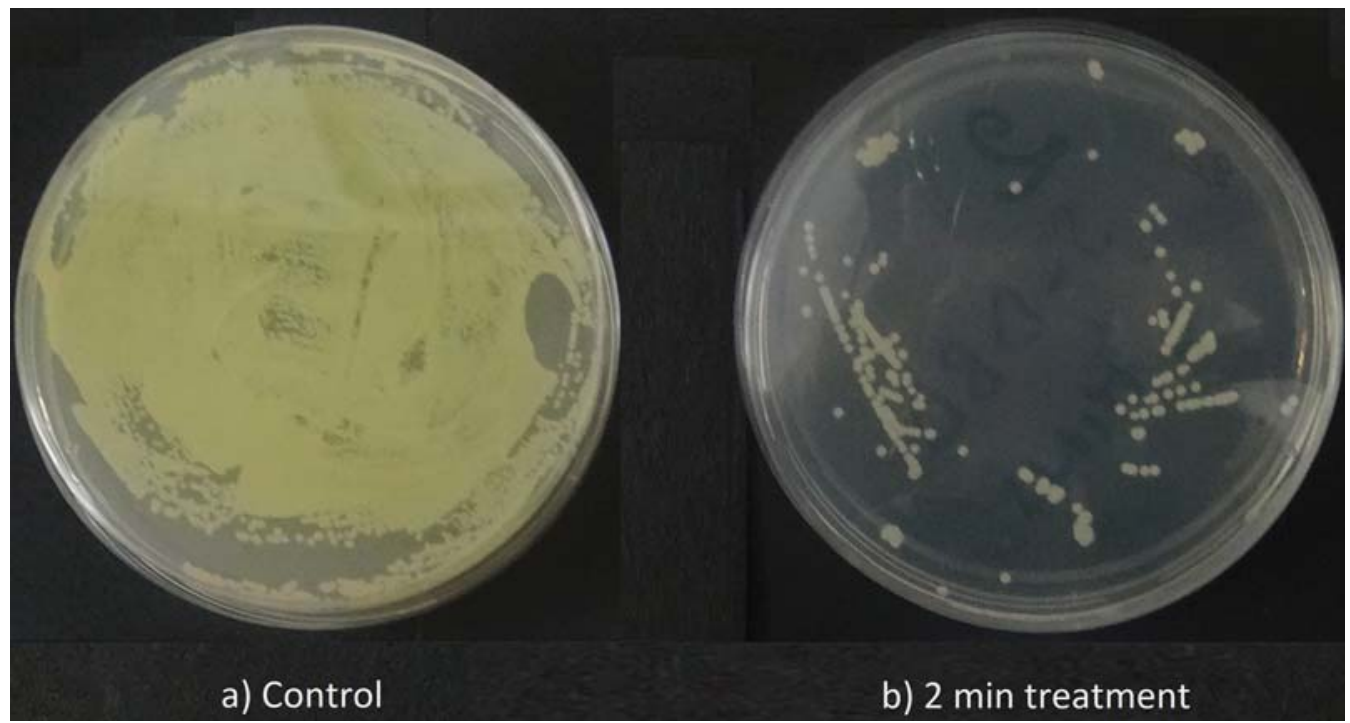

FIG. 6: $C$. guilliermondii proliferation on agar substrate in a control sample (a) and after $2 \mathrm{~min}$ of treatment (b) 
effect is clearly visible in the inactivation effect over agar substrate after 2 min of treatment. By considering the Petri dish area, a plasma average power of $14 \mathrm{~W}$, a $50 \%$ duty cycle and the treatment time of $5 \mathrm{~min}$, allowing a $5 \log$ CFU reduction, it is possible to state that a specific energy dose of $33 \mathrm{~J} / \mathrm{cm}^{2}$ is needed for a reliable sterilization degree. For the treatment in water solution, the spreading effect of the jet could be less important because species hitting the surface are then diluted within the whole liquid volume.

Inactivation results already shown, demonstrate that the PSJA presented in this work is effective against $C$. guilliermondii. Moreover, the inactivation efficacy is enhanced when charges advected by the induced flow are left free to reach the sample to be treated. The presence of these charges can eventually trigger chemical reactions responsible for the additional inactivation potential.

To complete the characterization of the plasma source, we quantified the species produced by the plasma into water exposed to the treatment for different exposure times. All of the results of these measurements are collected in Fig. 7.

First, we measured the $\mathrm{pH}$ of the treated solutions and the nitrate ion concentration by ion chromatography (Fig. 7a). We did not detect any signal due to nitrite ions. The $\mathrm{pH}$ of the solution drops by increasing the plasma treatment time, reaching a value of about 3 after $15 \mathrm{~min}$. At the same time, a linear increase of the concentration of nitrate ions is observed. The concentration of $\mathrm{H}_{3} \mathrm{O}^{+}$and of $\mathrm{NO}_{3}^{-}$ions increase consistently, reaching a value of about 1.2-1.3 $\mathrm{mM}$ after $15 \mathrm{~min}$ treatment, suggesting that both derive by the dissociation of nitric acid in solution. ${ }^{50}$ We did not observe any significant difference in $\mathrm{pH}$ or nitrate ions concentration by connecting the metal grid to the ground or by leaving it at floating potential.

We used the methods proposed by Bader and Hoigné ${ }^{45}$ and Eisenberg ${ }^{46}$ to quantify ozone and hydrogen peroxide in solution, respectively (Fig. 7b,c). Ozone concentration increases rapidly during the first min of treatment, reaching a plateau value of about $11 \mu \mathrm{M}$. The concentration of hydrogen peroxide instead remains very low during the first 5 min of treatment and then increases rapidly following an approximately linear trend. After 15 min of treatment, we measured a hydrogen peroxide concentration of about $170 \mu \mathrm{M}$. The mechanisms of formation and decomposition of ozone and hydrogen peroxide in solution due to the application of a non-thermal plasma have already been discussed in previous works. ${ }^{4-50}$ As found for $\mathrm{pH}$ and nitrate ions, we did not observe any significant difference in the concentrations of ozone and hydrogen peroxide in experiments carried out with the metal grid connected to ground or maintained at floating potential.

Finally, using the method proposed by Newton, ${ }^{47}$ we measured the rate of formation of $\mathrm{OH}$ radicals in solution. This method exploits the reaction of coumarin-3-carboxylic acid (CCA) with $\mathrm{OH}$ radicals to form the highly fluorescent product 7-hydroxycoumarin carboxylic acid (7-CCA-OH) with a known yield of 4.7\%. By measuring the concentration of 7-CCA-OH as a function of treatment time (Fig. 7d) and by interpolating the data points at short reaction times with a straight line, it is possible to obtain the rate of formation of 7-CCA-OH and, consequently, considering the yield of the reaction, the rate of formation of $\mathrm{OH}$ radicals. We obtained a rate of formation of $\mathrm{OH}$ radicals equal

Volume 8, Issue 3, 2018 

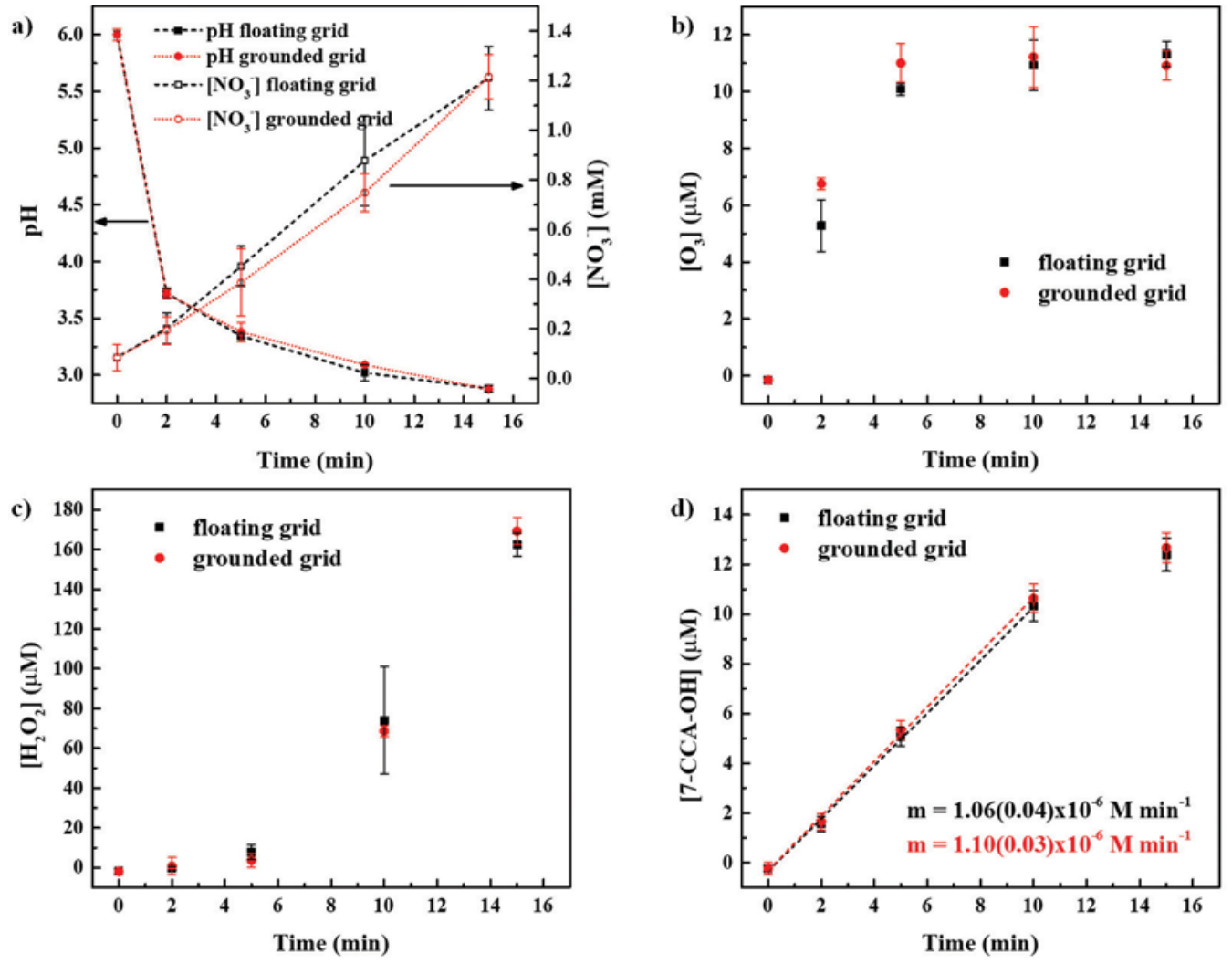

FIG. 7: Species produced in water by plasma treatment as a function of treatment time with the metal grid floating (black squares) or grounded (red circles). (a) $\mathrm{pH}$ and nitrate ions concentration; (b) concentration of ozone in solution; (c) concentration of hydrogen peroxide; (d) concentration of 7-CCA-OH (in these experiments, a CCA aqueous solution was used instead of pure water; see text for details). The dashed lines in (a) are just a guide for the eye; those in (d) are the interpolation of the first four points using straight lines.

to $(2.34 \pm 0.06) \cdot 10^{-5} \mathrm{M} \mathrm{min}^{-1}$ when the metal grid was connected to ground and $(2.26 \pm$ $0.09) \cdot 10^{-5} \mathrm{M} \mathrm{min}^{-1}$ when it was left at floating potential.

\section{CONCLUSIONS}

The ability of a PSJA to inactivate $C$. guilliermondii both in agar substrate and saline solution has been experimentally studied. The influence on the inactivation process of charged particles advected by the induced flow has been investigated as well. These charges have been blocked or left free to reach the sample by interposing a metal mesh between the PSJA and Petri dish surface. When the mesh is left floating, charge distribution onto the target surface is slightly modified with respect the condition without the mesh. When the mesh is connected to the ground, only $10 \%$ of the charge reaches the sample. 
Treatments in agar substrate showed a $\log 3 \mathrm{CFU}$ reduction after a $30 \mathrm{sec}$ treatment and a $\log 5 \mathrm{CFU}$ reduction after $5 \mathrm{~min}$. The spreading effect of the tubular jet impinging the Petri surface allows to homogeneously treat the whole sample with a specific energy dose of $33 \mathrm{~J} / \mathrm{cm}^{2}$ for the $\log 5 \mathrm{CFU}$ reduction. In saline solution, a log $3 \mathrm{CFU}$ reduction was obtained after $15 \mathrm{~min}$ of treatment. Charged particles advected by the induced jet lead to an additional log 1 CFU reduction for the longest treatment times, pointing out a dependence on energy dose of the chemical processes taking place in the treated sample and triggered by the presence of charges.

The analysis of water subjected to plasma treatment enabled us to determine and quantify a few important reactive species formed in solution that may be responsible for the observed biological inactivation effects, notably $\mathrm{OH}$ radicals, ozone, and hydrogen peroxide. In addition, we also measured the solution $\mathrm{pH}$ and the concentration of nitrate ions. The results of all of these measurements show no appreciable differences due to the experimental configuration used; that is, with the metal grid at floating potential or grounded. These results indicate that, under our conditions, the main ways of formation of the species that we quantified do not involve the charged species present in the air plasma, only neutrals. Specifically, it can be deduced that the $\mathrm{H}_{3} \mathrm{O}^{+}$that is detected in the liquid phase is formed mainly by the dissociation in water of nitric acid formed from NOx according to reactions described elsewhere. ${ }^{48-50}$ This conclusion, which is supported by the matching concentrations of $\mathrm{H}_{3} \mathrm{O}^{+}$and $\mathrm{NO}_{3}^{-}$determined in solution, suggests that there is not major contribution due to direct transfer of $\mathrm{H}_{3} \mathrm{O}^{+}$ from the gas into the liquid phase. Hydronium ion and its water clusters, $\mathrm{H}_{3} \mathrm{O}^{+}\left(\mathrm{H}_{2} \mathrm{O}_{n}\right.$, were indeed shown previously to be the major long-lived positive ions in air non thermal plasma). ${ }^{51-55}$ It cannot be excluded, however, that these positive ions present in the air plasma interact directly with the Candida cells on the surface of the medium, saline solution, or agar without dissolving into the medium in the absence of the cells. An alternative hypothesis considers that other ionic species or species derived by ionic reactions that have not yet been identified and quantified are involved to produce the observed biological effects of nonthermal plasma. Therefore, further studies must be carried out to investigate and understand the nature and the role of charged particles in the interaction with biological samples.

In conclusion, this work shows the efficacy of this indirect plasma treatment and the advantage of combining together fluid dynamics and inactivation properties of DBDs. Directional effects, scalability, and presence of charged particles enhancing inactivation effects are main features of this type of reactor. The efficacy of this plasma reactor against C. guilliermondii opens new perspectives to control this pathogen, widely diffuse in nature and able to induce diseases in immunocompromised humans and animals, with the ability to express a natural resistance to some drugs. Further studies must be carried out to understand the role of charged particles on the sample and the processes involved in bringing about the inactivation effects. A parallel line of development will focus on increasing the amount of advected charges in an attempt to further enhance the treatment efficacy. Lastly, new inactivation treatments could include $C$. albicans, the pathogenic role of which is improved by biofilm production on host tissues and medical device.

Volume 8, Issue 3, 2018 


\section{REFERENCES}

1. Pfaller MA, Andes DR, Diekema DJ, Horn DL, Reboli AC, Rotstein C, Franks B, Azie NE. Epidemiology and outcomes of invasive Candidiasis due to non-albicans species of Candida in 2,496 patients: data from the Prospective Antifungal Therapy (PATH) registry 2004-2008. PLoS One. 2014;9(7):e101510.

2. Tortorano AM, Peman J, Bernhardt H, Klingspor L, Kibbler CC, Faure O, Biraghi E, Canton E, Zimmermann K, Seaton S, Grillot R. The ECMM Working Group on Candidaemia. Epidemiology of Candidaemia in Europe: results of 28-month European Confederation of Medical Mycology (ECMM) hospital-based surveillance study. Eur J Clin Microbiol Infect Dis. 2004;23(4):317-22.

3. Savini V, Catavitello C, Onofrillo D, Masciarelli G, Astolfi D, Balbinot A, Febbo F, D'Amario C, D'Antonio D. What do we know about Candida guilliermondii? A voyage throughout past and current literature about this emerging yeast. Mycoses. 2011;54(5):434-41.

4. Cleff MB, de Lima AP, de Faria RO, Mano Meinerz AR, de Ávila Antunes T, Biasoli de Araújo F, da Silva Nascente P, de Oliveira NobreII M, MeirelesI MCA. Isolation of Candida spp from vaginal microbiota of healthy canine females during estrous cycle. Brazilian J Microbiol. 2005;36(2):201-4.

5. Pfaller MA, Diekema DJ, Mendez M, Kibbler C, Erzsebet P, Chang SC, Gibbs DL, Newell VA. The global antifungal surveillance group. Candida guilliermondii, an opportunistic fungal pathogen with decreased susceptibility to fluconazole: geographic and temporal trends from the ARTEMIS DISK antifungal surveillance program. J Clin Microbiol. 2006;44(10):3551-6.

6. Barchiesi F, Spreghini E, Tomassetti S, Della Vittoria A, Arzeni D, Manso E, Scalise G. Effects of caspofungin against Candida guilliermondii and Candida parapsilosis. Antimicrob Agents Chemother. 2006;50(8):2719-27.

7. Fridman G. Applied plasma medicine. Plasma Processes Polymers. 2007;5(6):503-33.

8. Bourke P, Ziuzina D, Han L, Cullen PJ, Gilmore BF. Microbiological interactions with cold plasma. J Appl Microbiol. 2017;123(2):308-24.

9. Ying J, Chunsheng R, Zhilong X, Dezhen W, Younian W, Hong Y. Comparison of yeast inactivation treated in He, Air and N2 DBD plasma. Plasma Sci Technol. 2006;8(6):720-723.

10. Aboubakr HA, Gangal U, Youssef MM, Goyal SM, Bruggeman PJ. Inactivation of virus in solution by cold atmospheric pressure plasma: identification of chemical inactivation pathways. J Phys D Appl Phys. 2016;49:204001.

11. Neretti G, Morandi B, Taglioli M, Poglayen G, Galuppi G, Tosi G, Borghi CA. Inactivation of Eimeria oocysts in aqueous solution by a dielectric barrier discharge plasma in contact with liquid. $\mathrm{J}$ Plasma Med. 2018;8(2):155-162.

12. Koban I, Matthes R, Hubner NO, Welk A, Meisel P, Holtfreter B, Sietmann R, Kindel E, Weltmann $\mathrm{KD}$, Kramer A. Treatment of Candida albicans biofilms with low-temperature plasma induced by dielectric barrier discharge and atmospheric pressure plasma jet. New J Phys. 2010;(7):073039.

13. Moravský L, Klas M, Machová E, Pisklová K, Matejčík S. Influence of a plasma jet on the viability of Candida albicans. Open Chem. 2015;13:257-62.

14. Ying S, Dongping L, Longfei J, Wenchun W, Jinhai N, Xianhui Z. Plasma inactivation of Candida albicans by an atmospheric cold plasma brush composed of hollow fibers. IEEE Trans Plasma Sci. 2012;40(4):1098-1102.

15. Doria ACOC, Sorge CDPC, Santos TB, Brandao J, Goncalves PAR, Maciel HS, Khouri S, Pessoa RS. Application of post-discharge region of atmospheric pressure argon and air plasma jet in the contamination control of Candida albicans biofilms. Res Biomed Eng. 2015;31:358-62.

16. Koban I, Matthes R, Hubner NO, Welk A, Meisel P, Holtfreter B, Holtfreter B, Sietmann R, Kindel E, Weltmann KD, Kramer A, Kocher T. Treatment of Candida albicans biofilms with low temperature plasma induced by dielectric barrier discharge and atmospheric pressure plasma jet. New J Phys. 2010;12:73039. 
17. Maisch T, Shimizu T, Isbary G, Heinlin J, Karrer S, Klampf TG, Li Y-G, Morfill G, Zimmermann JL. Contact-free inactivation of Candida albicans biofilms by cold atmospheric air plasma. Appl Environ Microbiol. 2012;78(12):4242-7.

18. Shi X-M, Zhang G-J, Yuan Y-K, Ma Y, Xu G-M, Yang Y. Research on the inactivation effect of lowtemperature plasma on Candida albicans. IEEE Trans Plasma Sci. 2008;36(2):498-503.

19. Sun Y, Yu S, Sun P, Wu H, Zhu W, Liu W, Zhang J, Fang J, Li R. Inactivation of Candida biofilms by non-thermal plasma and its enhancement for fungistatic effect of antifungal drugs. PLoS One. 2012;7:e40629.

20. Fridman A, Kennedy LA. Plasma physics and engineering, 2nd ed. Boca Raton, FL: CRC Press; 2004.

21. Fridman A, Chirokov A, Gutsol A. Non-thermal atmospheric pressure discharges. J Physics D: Appl Phys. 2005;38(2):R1-R24.

22. Neretti G, Seri P, Taglioli M, Shaw A, Iza F, Borghi CA. Geometry optimization of linear and annular plasma synthetic jet actuators. J Phys D Appl Phys. 2016;50(1):015210.

23. Taglioli M, Shaw A, Wright A, FitzPatrick B, Neretti G, Seri P, Borghi CA, Iza F. EHD-driven mass transport enhancement in surface dielectric barrier discharges. Plasma Sources Sci Technol. 2016;25(6):06LT01.

24. Corke TC, Post ML, Orlov DM. SDBD plasma enhanced aerodynamics: concepts, optimization and applications. Progress Aerospace Sci. 2007;43(7-8):193-217.

25. Dragonas FA, Neretti G, Sanjeevikumar P, Grandi G. High-voltage high-frequency arbitrary waveform multilevel generator for DBD plasma actuators. IEEE Trans Industry Appl. 2015;51(4):3334-42.

26. Moreau E. Airflow control by non-thermal plasma actuators. J Phys D Appl Phys. 2007;40(3):605-36.

27. Orlov DM, Font GI, Edelstein D. Characterization of discharge modes of plasma actuators. AIAA J. 2008;46(12):3142-8.

28. Santhanakrishnan A, Jamey DJ. Flow control with plasma synthetic jet actuators. J Phys D Appl Phys. 2007;40(3):637-651.

29. Neretti G. Active flow control by using plasma actuators. In: Recent Progress in Some Aircraft Technologies. Agarwal R, editor. InTech Open; 2016.

30. Maden I, Maduta R, Kriegseis J, Jakirlić S, Schwarz C, Grundmann S, Tropea C. Experimental and computational study of the flow induced by a plasma actuator. Int J Heat Fluid Flow. 2013;41:80-9.

31. Borghi CA, Cristofolini A, Neretti G, Seri P, Rossetti A, Talamelli A. Duty cycle and directional jet effects of a plasma actuator on the flow control around a NACA0015 airfoil. Meccanica. 2017; 52(2):1-14.

32. Kurz A, Grundmann S, Tropea C, Forte M, Seraudie A, Vermeersch O, Arnal D, Goldin R, King R. Boundary layer transition control using DBD plasma actuator. AerospaceLab. 2013;XX:1-8.

33. Shang JS, Kimmel RL, Menart JA, Sergey TS. Hypersonic flow control using surface plasma actuator. J Propul Power. 2008;24(5):923-34.

34. Junhui H, Corke TC, Flint OT. Plasma actuators for separation control of low-pressure turbine blades. AIAA J. 2006;44(1):51-7.

35. Van Ness DK, Corke TC, Morris SC. Tip clearance control using plasma actuators. Proceedings of the 44th AIAA Aerospace Sciences Meeting Exhibit; 2006 Jan. 9-12; Reno, NV. Paper AIAA-2006-21.

36. Cristofolini A, Borghi CA, Neretti G. Charge distribution on the surface of a dielectric barrier discharge actuator for the fluid-dynamic control. J Appl Phys. 2013;113(14):073303.

37. Cristofolini A, Borghi CA, Neretti G. Effect of the charge surface distribution on the flow field induced by a dielectric barrier discharge actuator. J Appl Phys. 2013 Apr 10.

38. Neretti G, Ricchiuto AC, Borghi CA. Measurement of the charge distribution deposited by an annular plasma synthetic jet actuator over a target surface. J Phys D Appl Phys. 2018;51(32):324004.

39. Fridman G, Brooks AD, Balasubramanian M, Fridman A, Gutsol A, Victor N, Ayan H, Friedman G. Comparison of direct and indirect effects of non-thermal atmospheric-pressure plasma on bacteria. Plasma Process Polym. 2007;4(4):370-5.

Volume 8, Issue 3, 2018 
40. Sysolyatina E, Mukhachev A, Yurova M, Grushin M, Karalnik V, Petryakov A, Trushkin N, Ermolaeva S, Akishev Y. Role of the charged particles in bacteria inactivation by plasma of a positive and negative corona in ambient air. Plasma Process Polym. 2014;11(4):315-34.

41. Laroussi M. Mechanisms of interaction of cold plasma with bacteria. AIP Conference Proceedings. 2008; 1041.

42. Dobrynin D, Friedman G, Fridman A, Starikovski A. Inactivation of bacteria using dc corona discharge: role of ions and humidity. New J Phys. 2011;13:103033.

43. Neretti G, Cristofolini A, Borghi CA. Experimental investigation on a vectorized aerodynamic dielectric barrier discharge plasma actuator array. J Appl Phys. 2014;15(16).

44. Meeker DC. Finite element method magnetics, version 4.2. Available from: http://femm.info.

45. Bader H, Hoigné J. Determination of ozone in water by the indigo method. Water Res. 1981;15(4):44956.

46. Eisenberg G. Colorimetric determination of hydrogen peroxide. Ind Eng Chem Anal Ed. 1943;15(5):327-8.

47. Newton GL, Milligan JR. Fluorescence detection of hydroxyl radicals. Radiat Phys Chem. 2006;75(4):473-8.

48. Bosi FJ, Tampieri F, Marotta E, Bertani R, Pavarin D, Paradisi C. Characterization and comparative evaluation of two atmospheric plasma sources for water treatment. Plasma Process Polym. 2018;15(3):1700130.

49. Tampieri F, Giardina A, Bosi FJ, Pavanello A, Marotta E, Zaniol B, Neretti G, Paradisi C. Removal of persistent organic pollutants from water using a newly developed atmospheric plasma reactor. Plasma Process Polym. 2018;15(6):1700207.

50. Nani L, Tampieri F, Ceriani E, Marotta E, Paradisi C. A versatile prototype plasma reactor for water treatment supporting different discharge regimes. J Phys D Appl Phys. 2018;51(27):274001.

51. Pandis SN, Seinfeld JH, Geophys J. Sensitivity analysis of a chemical mechanism for aqueous-phase atmospheric chemistry. J Geophys Res. 1989;94:1105-26.

52. Donó A, Paradisi C, Scorrano G. Abatement of volatile organic compounds by corona discharge. A study of the reactivity of trichloroethylene under atmospheric pressure ionization conditions. Rapid Comm Mass Spectrometry. 1997;11(15):1687-94.

53. Schiorlin M, Marotta E, Rea M, Paradisi C. Comparison of toluene removal in air at atmospheric conditions by different corona discharges. Environ Sci Technol. 2009;43(24):9386-92.

54. Marotta E, Paradisi C. A mass spectrometry study of alkanes in air plasma at atmospheric pressure. J Amer Soc Mass Spectrometry. 2009;20(4):697-707.

55. Marotta E, Callea A, Ren X, Rea M, Paradisi C. DC corona electric discharges for air pollution control, 2 -ionic intermediates and mechanisms of hydrocarbon processing. Plasma Process Polym. 2008;5(2):146-54. 